\title{
Real-time optoacoustic monitoring of temperature in tissues
}

\author{
Irina V Larina ${ }^{1,2}$, Kirill V Larin ${ }^{1,4}$ and Rinat O Esenaliev ${ }^{1,2,3,5}$ \\ ${ }^{1}$ Center for Biomedical Engineering, University of Texas Medical Branch, Galveston, \\ TX 77555-0456, USA \\ 2 Department of Neuroscience and Cell Biology, University of Texas Medical Branch, \\ Galveston, TX 77555-0456, USA \\ ${ }^{3}$ Department of Anesthesiology, University of Texas Medical Branch, Galveston, \\ TX 77555-0456, USA \\ E-mail: rinat.esenaliev@utmb.edu
}

Received 30 November 2004, in final form 4 February 2005

Published 22 July 2005

Online at stacks.iop.org/JPhysD/38/2633

\begin{abstract}
To improve the safety and efficacy of thermal therapy, it is necessary to map tissue temperature in real time with submillimetre spatial resolution. Accurate temperature maps may provide the necessary control of the boundaries of the heated regions and minimize thermal damage to surrounding normal tissues. Current imaging modalities fail to monitor tissue temperature in real time with high resolution and accuracy. We investigated a non-invasive optoacoustic method for accurate, real-time monitoring of tissue temperature during thermotherapy. In this study, we induced temperature gradients in tissue and tissue-like samples and monitored the temperature distribution using the optoacoustic technique. The fundamental harmonic of a Q-switched Nd: YAG laser $(\lambda=1064 \mathrm{~nm})$ was used for optoacoustic wave generation and probing of tissue temperature. The tissue temperature was also monitored with a multi-sensor temperature probe inserted in the samples. Good agreement between optoacoustically measured and actual tissue temperatures was obtained. The accuracy of temperature monitoring was better than $1{ }^{\circ} \mathrm{C}$, while the spatial resolution was about $1 \mathrm{~mm}$. These data suggest that the optoacoustic technique has the potential to be used for non-invasive, real-time temperature monitoring during thermotherapy.
\end{abstract}

\section{Introduction}

Non-invasive real-time measurement of temperature distribution in tissues during thermotherapy is necessary for the safe and efficient thermal destruction of tumours or other abnormal tissues (Lehmann 1990, Welch and van Gemert 1995). Wide clinical application of thermotherapy is limited due to the lack of temperature control over the heated volume. Safe and efficient thermotherapy requires real-time temperature measurements with $1 \mathrm{~mm}$ spatial resolution and $1^{\circ} \mathrm{C}$ or better accuracy.

Infrared thermography is capable of real-time temperature measurements with an accuracy of $0.1^{\circ} \mathrm{C}$. However, this

\footnotetext{
4 Present address: Biomedical and Mechanical Engineering Department, University of Houston, Houston, TX 77204-4006, USA.

5 Author to whom any correspondence should be addressed.
}

technique can measure temperature only from superficial tissue layers (Welch and van Gemert 1995). Ultrasound methods can provide real-time images with good resolution and depth of penetration, but they lack sufficient accuracy for temperature measurements (Seip and Ebbini 1995, MaassMoreno and Damianou 1996, Seip et al 1996). MRI requires a long acquisition time to provide temperature mapping with high resolution and accuracy (Graham et al 1998, Steiner et al 1998).

The laser optoacoustics (OA) technique has been developed and applied in many areas of biomedicine (Karabutov et al 2003, Wang 2003). For example, the OA method was successfully applied for breast cancer imaging (Esenaliev et al 1999, Kirillov et al 1999, Wang et al 2002, Andreev et al 2003, Copland et al 2004), functional and structural imaging of the brain (Wang et al 2003a, 2003b, 


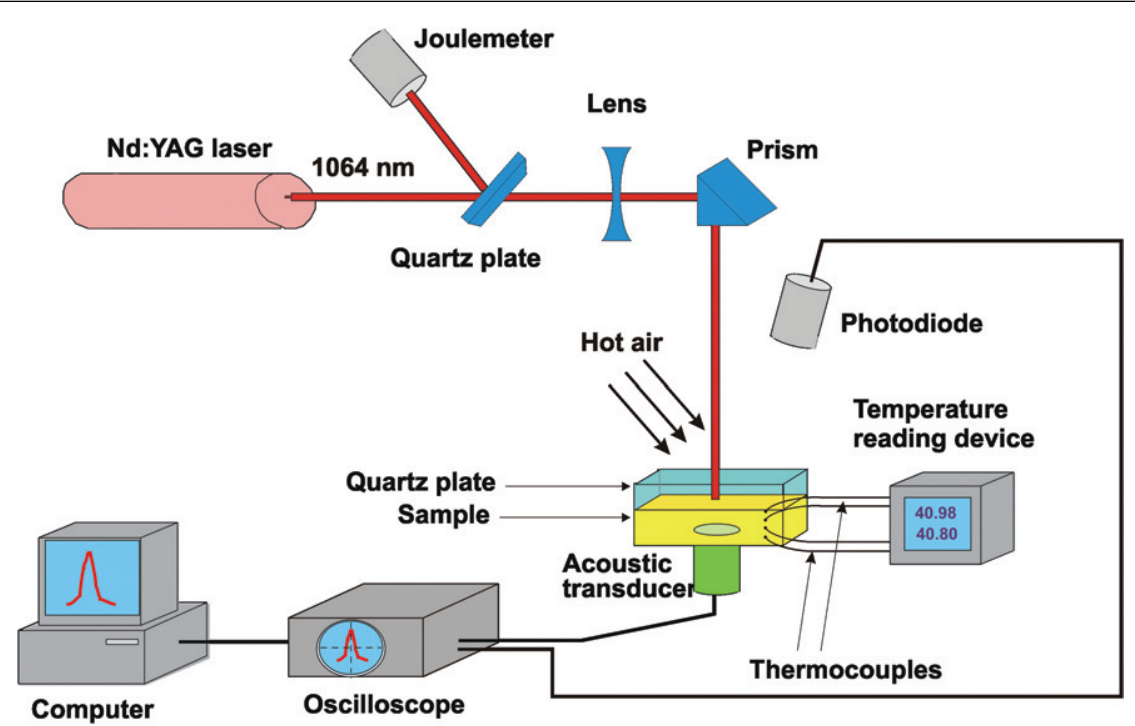

Figure 1. Experimental set-up for optoacoustic measurements of temperature in tissue and tissue phantoms.

(This figure is in colour only in the electronic version)

2004), and real-time monitoring of oxygenation (Esenaliev et al 2002) and total haemoglobin concentration (Esenaliev et al 2004). Recently, we used the OA technique to monitor the cooling and freezing of tissues (Larin et al 2002); this can be applied for precise cryotherapy. All these applications utilize differences in optical properties between normal and modified tissue states.

That the OA technique is capable of non-invasive temperature determination of fungus of the eye during laser irradiation has recently been demonstrated in (Schule et al 2004). It has been shown that time-resolved detection and analysis of the laser-induced pressure profiles allow for reconstruction of $\mathrm{OA}$ images and calculation of tissue temperature with an accuracy of about $17 \%$. In this paper, we study the capability of the OA technique to monitor the temperature distribution deeply in strongly scattering tissues and tissue phantoms, at temperatures typical for hyperthermia.

\section{Materials and methods}

\subsection{Experimental set-up}

The experimental set-up used in this study is shown in figure 1. The fundamental $(\lambda=1064 \mathrm{~nm})$ harmonic of a Q-switched nanosecond Nd: YAG laser was used for optoacoustic wave generation. A diverging lens was used to provide a wide $(10 \mathrm{~mm}$ in diameter) laser spot on the sample. The energy of each laser pulse was measured with a calibrated joulemeter. The energy of incident laser pulses was $15 \mathrm{~mJ}$, which yielded an incident laser fluence of $19 \mathrm{~mJ} \mathrm{~cm}^{-2}$. The pulsed laser radiation with such parameters induced an insignificant temperature rise $\left(<10^{-2}{ }^{\circ} \mathrm{C}\right)$ in the samples. The pulsed laser radiation was delivered to the samples from above with the use of a prism. In the experiments with aqueous, absorbing solutions of potassium chromate we used the third harmonic of the Nd: YAG laser $(\lambda=355 \mathrm{~nm})$.
Wide-band PVDF acoustic transducers $(0.1-5 \mathrm{MHz})$ were used for OA pressure wave detection. Although the transducers were heated, the sensitivity of the PVDF piezoelectric film does not depend on the temperature that yields accurate $\mathrm{OA}$ pressure wave measurements.

A photodiode (Det 210, Thorlabs, Inc.) was used to monitor the total diffuse reflectance $\left(R_{\mathrm{d}}\right)$ from samples. Since the photodiode was positioned at a large distance from the sample, the photodiode signal was proportional to $R_{\mathrm{d}}$.

The averaging and recording time for one signal was $1 \mathrm{~s}$. The repetition rate of the pulsed laser radiation was $10 \mathrm{~Hz}$ and allowed for averaging of 10 pressure wave profiles during this time. The pressure profiles were recorded by a digital scope (TDS 520, Tektronix Inc.) and in a computer.

Tissue heating was performed using hot air from a heatgun (figure 1). Tissue samples were placed on the transducer and covered by a quartz plate of diameter $40 \mathrm{~mm}$ and thickness $10 \mathrm{~mm}$ to avoid direct contact with hot air.

We performed slow and rapid heating of the samples by varying the distance between the heatgun and the sample surface. Slow heating $\left(\approx 2^{\circ} \mathrm{C} \mathrm{min}^{-1}\right)$ produced a uniform temperature distribution in the samples and was used to monitor changes in optical properties during heating. Rapid tissue heating by hot air $\left(\approx 1^{\circ} \mathrm{Cs}^{-1}\right)$ was used to obtain a one-dimensional temperature distribution (gradient) in tissue. Conductive heating through the quartz plate provided a plane temperature front in the sample parallel to the transducer surface. Tissue samples were also covered with a plastic film from other sides to avoid dehydration and lateral heating.

A multi-sensor temperature probe with a variable number of thermocouples (from two to four), connected to a temperature reading device (Scanner Plus, Azonix Inc.), was inserted into the samples to provide temperature measurements simultaneously at different depths with an accuracy of about $0.3^{\circ} \mathrm{C}$. The use of the multi-sensor probe allowed for reconstruction of the actual temperature gradient, which was compared with the OA temperature mapping. 


\subsection{Samples}

The experiments were performed with absorbing aqueous solutions of potassium chromate $\left(\mathrm{K}_{2} \mathrm{CrO}_{4}\right)$, gelatin phantoms, and freshly excised canine liver (five samples) and myocardium (five samples). The tissue samples had dimensions of about $50 \times 50 \mathrm{~mm}^{2}$ and a thickness of $10-30 \mathrm{~mm}$.

The aqueous solution of potassium chromate served as a model object with thermophysical properties close to that of pure water, but with absorption in the UV. Distilled water for solution preparation was boiled for $15 \mathrm{~min}$ to avoid air bubble formation during the heating. The absorption coefficient of the solution calculated from the optoacoustic pressure profile by exponential fitting was $11 \mathrm{~cm}^{-1}$.

To simulate tissue, we used a gelatin phantom of cylindrical shape with a radius of $60 \mathrm{~mm}$ and a thickness of $30 \mathrm{~mm}$. India ink was used as an absorber in the phantoms to obtain an absorption coefficient of $4 \mathrm{~cm}^{-1}$, typical of tissues in the NIR spectral range (Welch and van Gemert 1995).

\subsection{Theoretical background}

The thermal expansion of an absorbing medium heated by a short laser pulse with an incident laser fluence, $F_{0}$, induces a pressure rise, $P(z)$, in the irradiated volume upon stressconfined irradiation conditions (Gusev and Karabutov 1993):

$$
P(z)=\left(\frac{\beta c_{\mathrm{s}}^{2}}{C_{\mathrm{p}}}\right) \mu_{\mathrm{a}} F(z)=\Gamma \mu_{\mathrm{a}} F(z)=\Gamma \mu_{\mathrm{a}} F_{0} \mathrm{e}^{\left(-\mu_{\mathrm{a}} z\right)},
$$

where $\beta\left[1^{\circ} \mathrm{C}^{-1}\right]$ is the thermal expansion coefficient, $c_{\mathrm{s}}$ the speed of sound, $C_{\mathrm{p}}\left[\mathrm{J} \mathrm{g}^{-1}{ }^{\circ} \mathrm{C}^{-1}\right]$ the heat capacity at constant pressure, $F(z)\left[\mathrm{J} \mathrm{cm}^{-2}\right]$ the laser fluence and $\mu_{\mathrm{a}}\left[\mathrm{cm}^{-1}\right]$ the absorption coefficient of the medium. The expression $\beta c_{\mathrm{s}}^{2} / C_{\mathrm{p}}$ in equation (1) represents the Grüneisen parameter, $\Gamma$ (dimensionless). The factor $\mathrm{e}^{\left(-\mu_{\mathrm{a}} z\right)}$ represents exponential attenuation of the optical radiation in the medium. Therefore, the OA pressure is dependent on $\Gamma$, the fluence, and the optical properties of the medium. Equation (1) is valid under the condition of stress-confinement when pressure relaxation is negligible during the heat deposition. The stress-confined condition can be achieved in tissues by using nanosecond optical pulses.

The Grüneisen parameter of water is temperature dependent (Burmistrova et al 1979, Sigrist 1986). These studies demonstrated the linear dependence of the OA pressure amplitude on temperature in water and, thus, can be expressed by an empirical equation:

$$
\Gamma=A+B T,
$$

where $A$ and $B$ are constants and $T$ is the temperature. One can rewrite equation (1) in the case of an absorbing medium as

$$
P(z)=(A+B T(z)) \mu_{\mathrm{a}} F_{0} \mathrm{e}^{\left(-\mu_{\mathrm{a}} z\right)}
$$

and in the case of strongly scattering media in deep (not in the subsurface) areas as

$$
P(z)=(A+B T(z)) k \mu_{\mathrm{a}} F_{0} \mathrm{e}^{\left(-\mu_{\mathrm{eff}} z\right)},
$$

where $T(z)$ is the temperature distribution in tissue, $k$ the parameter resulting from multiple scattering in tissue and is dependent on the absorption and scattering coefficients (Welch and van Gemert 1995) and $\mu_{\text {eff }}$ is the effective attenuation coefficient.

Rearranging equation (4), one can obtain

$$
T(z)=C+\frac{D \cdot P(z)}{P(z)_{T=T_{0}}},
$$

where $P(z)_{T=T_{0}}$ is the OA pressure profile recorded at the initial temperature $T_{0}$, and $C$ and $D$ are parameters that are dependent on tissue properties. Therefore, by recording and analysing the temporal OA pressure profile, one can reconstruct the temperature distribution during hyperthermia.

\section{Results}

Figure 2 shows typical OA pressure profiles recorded from the aqueous solution of potassium chromate from $25^{\circ} \mathrm{C}$ to $70^{\circ} \mathrm{C}$. The amplitude of the OA pressure signal recorded at the temperature of $70^{\circ} \mathrm{C}$ was approximately 2.8 times greater than that at the temperature of $25^{\circ} \mathrm{C}$. A noticeable difference between the amplitude of OA pressure profiles recorded at $37^{\circ} \mathrm{C}$ and $43^{\circ} \mathrm{C}$ was also detected. The temporal profiles did not change significantly during heating and cooling. The OA pressure amplitude induced in the canine liver versus temperature from $36^{\circ} \mathrm{C}$ to $50^{\circ} \mathrm{C}$, and the $\mathrm{OA}$ pressure amplitude induced in the canine myocardium versus temperature from $28^{\circ} \mathrm{C}$ to $55^{\circ} \mathrm{C}$ are shown in figures $3(a)$ and $(b)$, respectively. It is clearly seen that the amplitude increases linearly with the temperature. The relative increase in pressure amplitude was approximately $1.5 \%$ per degree centigrade, which results in about $9 \%$ increase in the liver tissue and $4 \%$ increase in the myocardium tissue heated from $37^{\circ} \mathrm{C}$ to $43^{\circ} \mathrm{C}$. This temperature rise is normally applied for hyperthermia. The optical properties of liver tissue were constant during heating, up to the temperature of coagulation $\left(50-55^{\circ} \mathrm{C}\right)$, and dramatically changed due to protein denaturation at higher temperatures (data not shown).

The Grüneisen parameter of water and the OA pressure amplitude induced in the aqueous solution are presented versus temperature in figure 4. The Grüneisen parameter of water was theoretically calculated from the data on the thermal expansion coefficient, speed of sound and heat capacity at

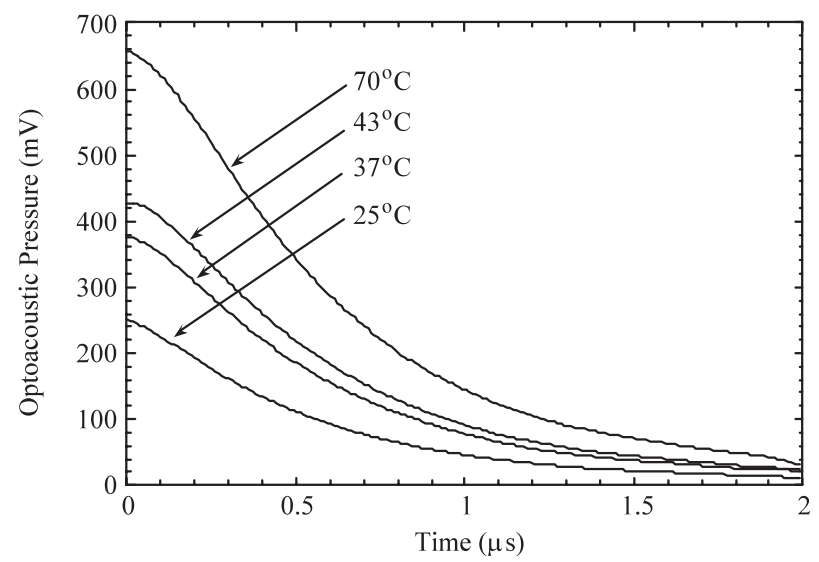

Figure 2. OA pressure profiles induced in aqueous solution at various temperatures. 

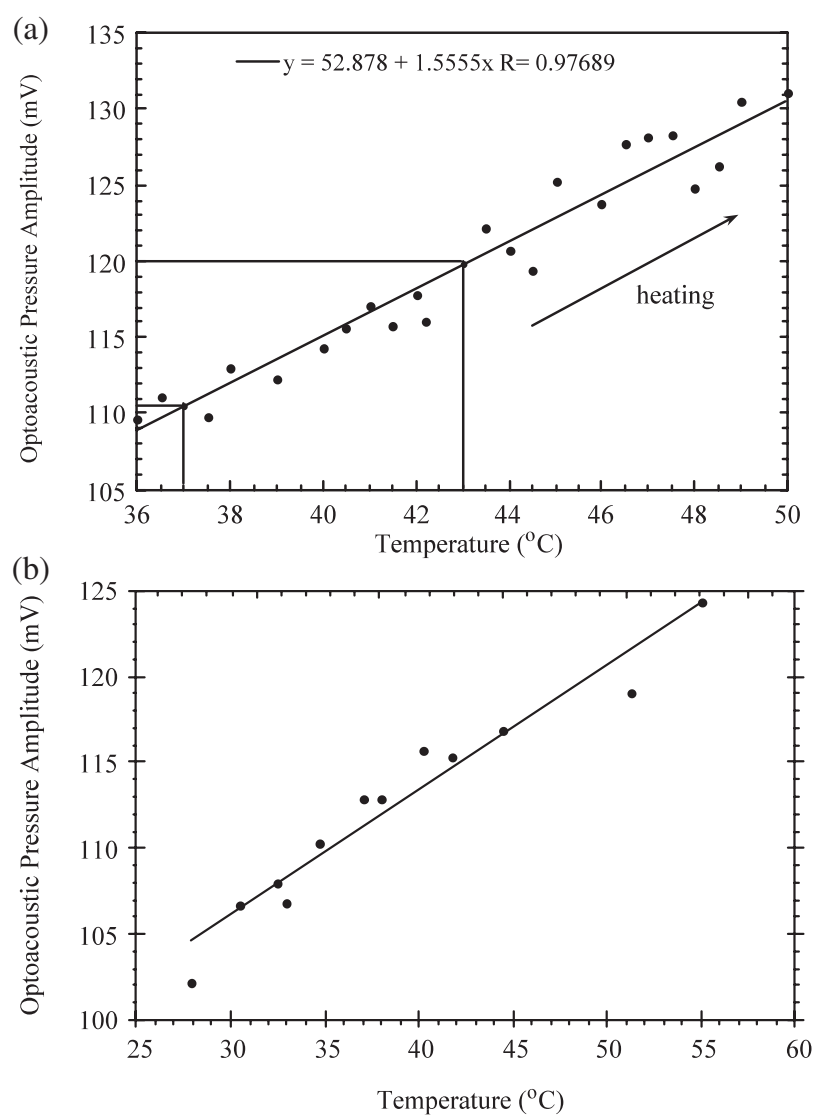

Figure 3. Amplitude of OA pressure induced in canine liver $(a)$ and canine myocardium $(b)$ versus temperature. The vertical and horizontal lines in $(a)$ mark the temperature range that is normally used in tumour hyperthermia.

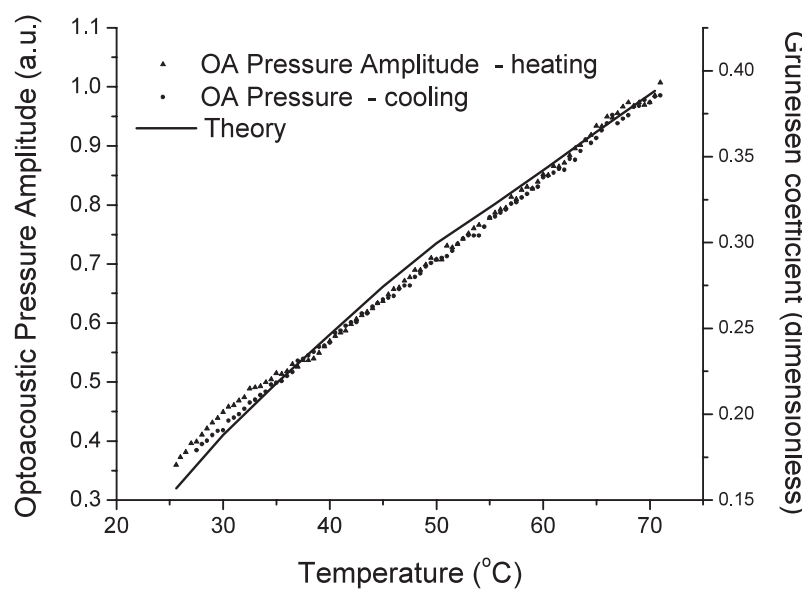

Figure 4. Theoretically calculated water Grüneisen coefficient and optoacoustic pressure amplitude induced in aqueous solution as a function of temperature.

constant pressure reported in Weast (1974) and Kikoin (1976). The Grüneisen coefficient has an almost linear dependence on temperature. There is a good correlation between the theoretical and experimental data.

Figure 5 presents the optical effective attenuation coefficient and total diffuse reflectance versus temperature recorded in the canine liver during slow conductive heating and passive cooling of the tissue samples (figures $5(a)$ and
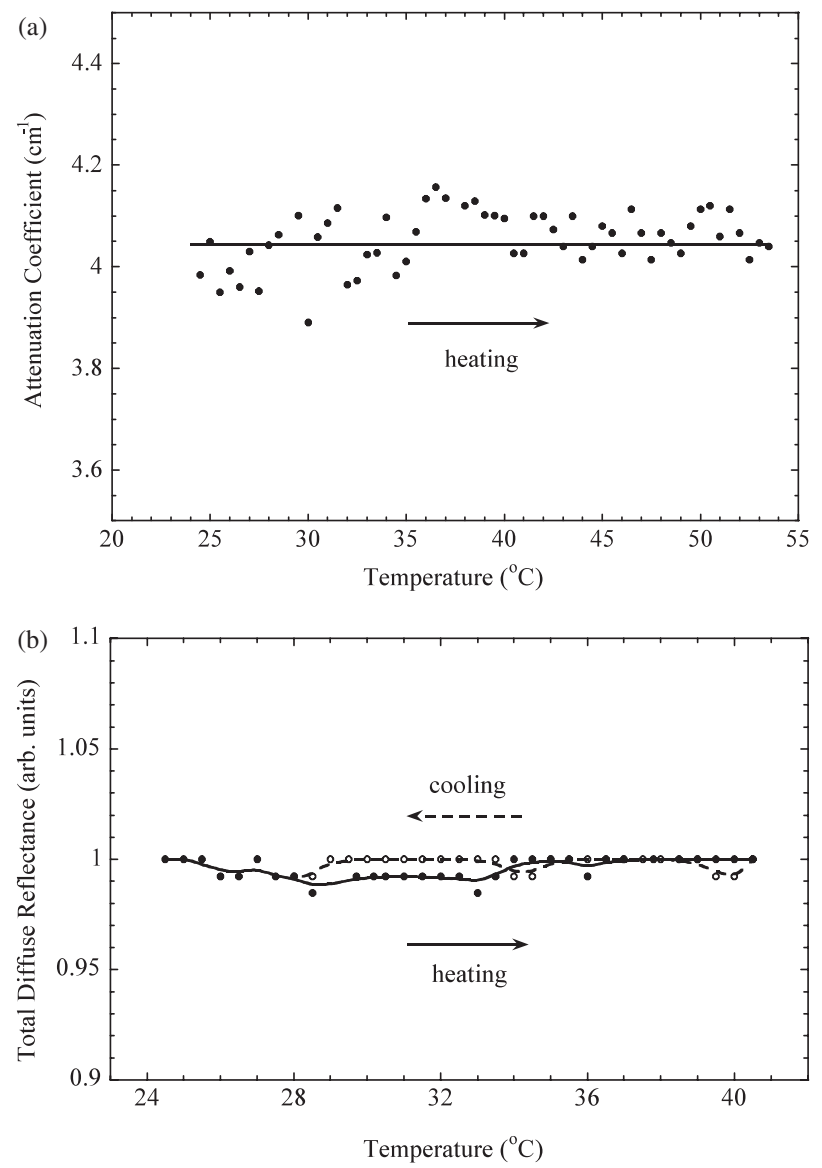

Figure 5. Optical attenuation coefficient ( $a$ ) and total diffuse reflectance $(b)$ versus temperature during slow conductive heating in canine liver. The solid line and solid circles indicate heating, while the dashed line and open circles correspond to the cooling of the tissue samples.

(b), respectively). No changes in the attenuation coefficient from $25^{\circ} \mathrm{C}$ to about $53^{\circ} \mathrm{C}$ were observed in these experiments. However, the OA pressure amplitude induced in the canine liver increased linearly with the temperature from $36^{\circ} \mathrm{C}$ to $50^{\circ} \mathrm{C}$ (figure 3(a)). Therefore, only the Grüneisen parameter of liver increased with temperature, while the optical properties did not change. These data experimentally confirm the hypothesis that the Grüneisen parameter of tissue increases with temperature.

OA pressure profiles used for temperature distribution reconstruction recorded at different times during fast heating of liver and gelatin samples are presented in figures $6(a)$ and $(b)$, respectively. Calibration graphs were plotted for liver and gelatin samples by using the temperature recorded at the sample surface by the first thermocouple of the multisensor temperature probe. The constants $C$ and $D$ were found from these graphs by fitting of $P(T)$ graphs for these samples. By using these constants and equation (5), we reconstructed temperature distributions from the recorded pressure profiles. The reconstructed temperature distributions in the liver and gelatin samples are shown in figures $7(a)$ and $(b)$, respectively. They were compared with the actual temperature recorded by the multi-sensor temperature probe (the circles indicate actual temperature readings from the multi-sensor temperature probe). The optoacoustically reconstructed temperature from figures $7(a)$ and $(b)$ versus 

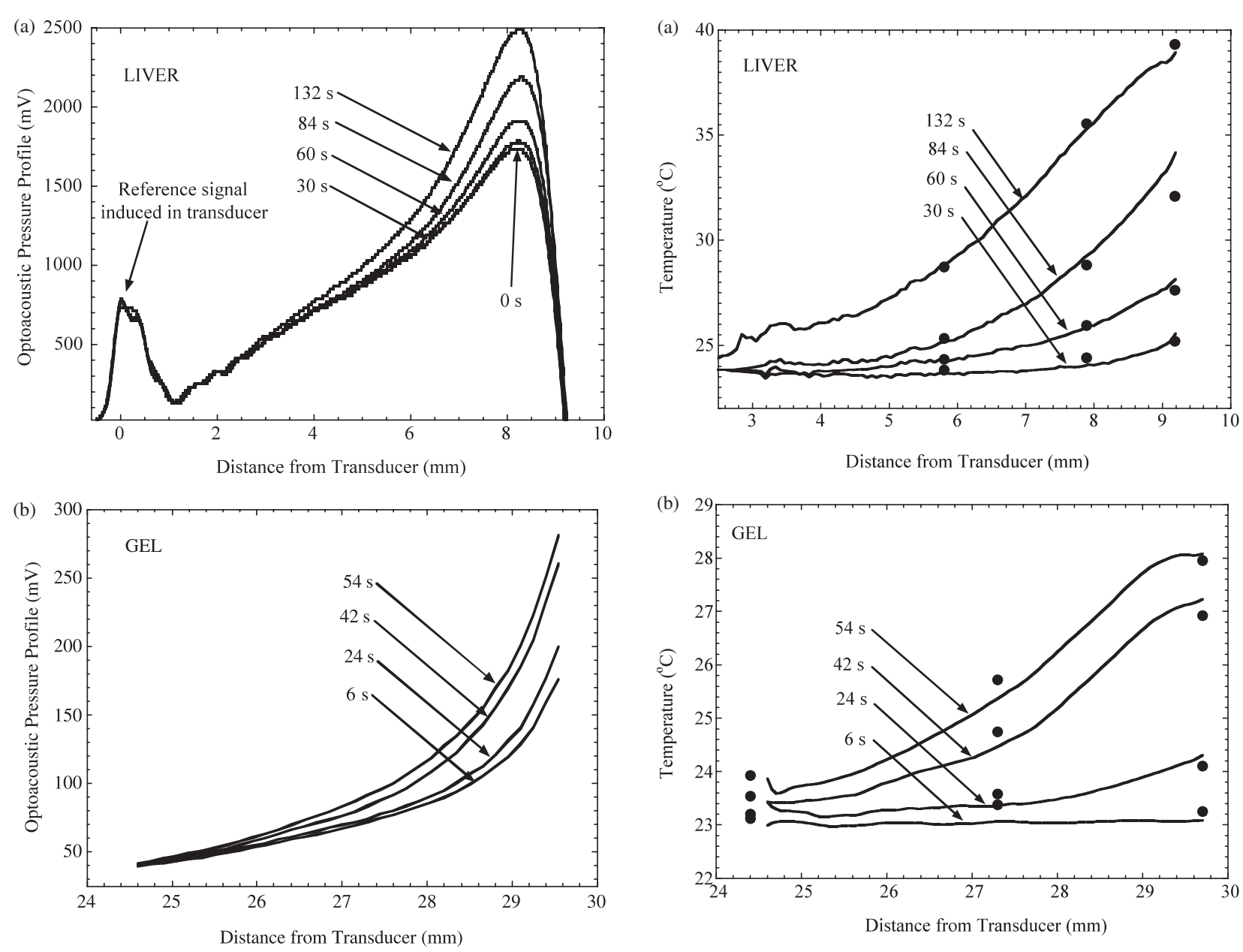

Figure 6. OA pressure profiles recorded from canine liver $(a)$ and gelatin phantom $(b)$ at different times during heating.

the actual temperature measured with thermocouples is shown in figure 8 . These results demonstrate good agreement between the temperature distributions reconstructed using the OA technique and those measured using the multi-sensor temperature probe. The accuracy of temperature monitoring was less than $1^{\circ} \mathrm{C}$, while the spatial resolution was about $1 \mathrm{~mm}$.

\section{Discussion}

Results of the experiment with the aqueous solution of potassium chromate demonstrated good agreement of the temperature dependence of the measured $\mathrm{OA}$ pressure amplitude with the Grüneisen parameter of water calculated theoretically from the literature data. The increase of the Grüneisen parameter of water with temperature is caused mostly by the increase in the thermal expansion coefficient $\beta$ (equation (1)). The slight deviation of the experimental data points from the theoretical curve observed in figure 4 can be explained by the differences between the thermophysical properties of the aqueous solution of potassium chromate and pure water.

The increase in the Grüneisen parameter of the investigated tissues with temperature is less than that in water. The difference in temperature dependence of the

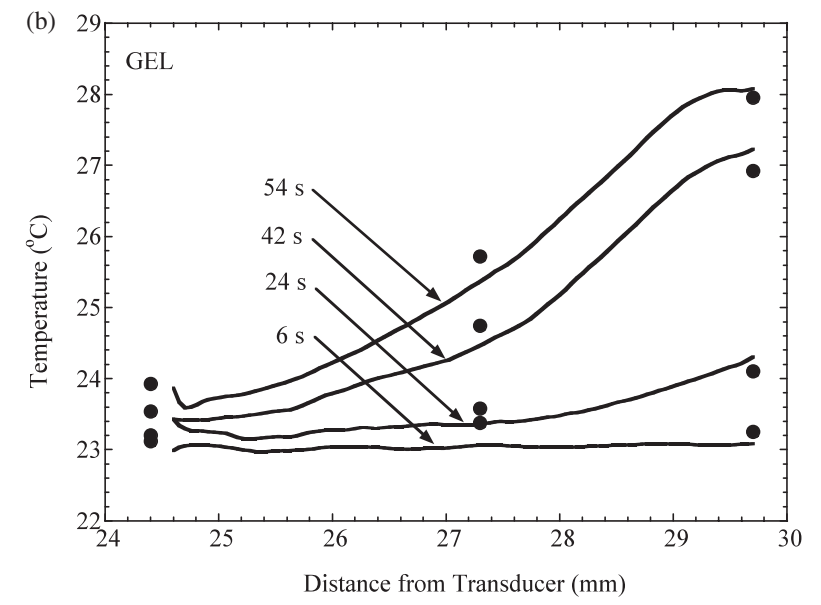

Figure 7. Reconstructed temperature profiles in canine liver $(a)$ and gelatin phantom $(b)$. Circles indicate actual temperature in the samples recorded with the multi-sensor temperature probe.

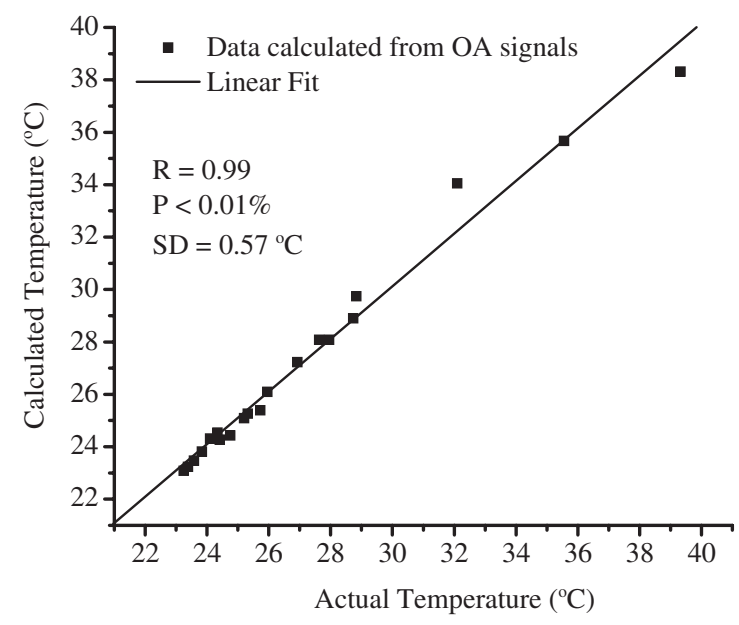

Figure 8. Temperature calculated from OA signals versus actual temperature measured with the multi-sensor temperature probe for data shown in figure 7: $R$-correlation coefficient: $P-p$-value; SD—standard deviation of data points.

Grüneisen parameters of water, liver and myocardium was due to the composition of the tissues. The tissue thermal expansion coefficient and speed of sound are less dependent on temperature than those corresponding to water (Duck 1990). 
The proportions of water, lipid and proteins vary in tissues, and this yields different thermal expansion coefficients, speeds of sound, and heat capacities, as well as different dependences of these parameters on temperature. It is likely that the lipid content is a major factor because the thermal expansion coefficient and speed of sound in fat decrease with temperature (Duck 1990). This explains the differences in the temperature dependence of the Grüneisen parameter of water, liver and myocardium, because the lipid content in liver and myocardial tissue is $4.6 \%$ and 6.2\%, respectively (Duck 1990).

The results obtained demonstrated that the attenuation coefficient and total diffuse reflectance of tissue do not change during moderate heating in the temperature range usually applied for hyperthermia. This means that absorption and reduced scattering coefficients do not significantly change in this temperature range.

Data from (Gertner et al 1997, Clarke et al 2003) suggest a slight decrease in the ultrasound attenuation coefficient (UAC) for temperatures under $50^{\circ} \mathrm{C}$ in tissues. Therefore, one could expect minimal influence of the temperature UAC dependence on the optoacoustic signals detected at temperatures of up to $50^{\circ} \mathrm{C}$. The temperature dependence of UAC can be taken into account for more precise OA measurements during thermotherapy.

Although some data reported in the literature (Laufer et al 1998) indicate that the tissue reduced scattering coefficient $\mu_{\mathrm{s}}^{\prime}$ may increase (about $0.5 \%$ per degree centigrade) for temperatures below $50^{\circ} \mathrm{C}$, no measurable changes in the effective attenuation coefficient and total diffuse reflectance were observed in our studies (figures $5(a)$ and $(b)$ ). This variation in results can be explained by the differences between the tissue types used.

The linear dependence of the OA pressure amplitude on temperature obtained in our experiments was used for temperature mapping in liver and gelatin samples. As a result, we were able to monitor the temperature change in tissues by detecting the pressure wave and comparing it with the pressure wave recorded before the heating. An accurate reconstruction of temperature distribution in tissues requires calculation of the parameters $C$ and $D$ from equation (5). In general, these parameters can be calculated before or during thermotherapy. The parameters $C$ and $D$ could be calculated during thermotherapy by measuring the temperature at one point, for instance, on the tissue surface and comparing it with the pressure signal induced at this point. In our studies, we used one thermocouple placed on the sample surface for calibration. Another possible method of obtaining parameters $C$ and $D$ is to monitor the temperature from the surface using the infrared thermography technique.

The results shown in figures 7 and 8 indicate that one can monitor the temperature rise in tissues by detecting the pressure wave amplitude with an accuracy of better than $1^{\circ} \mathrm{C}$ at depths of up to $3 \mathrm{~cm}$ from the transducer surface. The standard deviation of data points was equal to $0.57^{\circ} \mathrm{C}$. The accuracy of tissue temperature measurements was limited in our experiments by the $3 \%$ instability of averaged (10 times) laser pulse energy. Current laser systems with stabilized pulse energy available in the market have $1 \%$ stability; therefore, a higher accuracy of temperature measurement could be achieved.

\section{Conclusion}

Our studies demonstrated that the OA technique can be used: (1) to detect temperature changes in tissue with an accuracy of better than $1^{\circ} \mathrm{C} ;(2)$ to perform temperature measurements in real time; (3) to provide temperature measurements at depths of up to several centimetres; and (4) to reconstruct the temperature distribution in tissues during heating. However, it is necessary to note that the studies in tissues were performed in vitro. The influence of different physiological parameters during tissue heating in vivo (such as increased tissue perfusion) on the accuracy of OA temperature monitoring should be evaluated in additional studies. However, one can expect a limited influence of these parameters, in vivo, on the accuracy of temperature monitoring with the OA technique at moderate (a few degrees) heating.

Since OA wave generation and detection are not influenced by the nature of the heating agent, this technique can potentially be applied for real-time temperature mapping in tissues during heating by radiofrequency, microwave, ultrasonic or laser radiation. In addition, optoacoustic monitoring is a non-invasive technique because low-energy laser pulses applied for pressure wave generation cannot cause thermal or mechanical damage to tissues. Therefore, optoacoustic monitoring of temperature has the potential to become a valuable real-time feed-back modality for thermotherapy.

\section{Acknowledgments}

The authors would like to thank Brent Bell for technical assistance and to acknowledge support from the Whitaker Foundation.

\section{References}

Andreev V G, Karabutov A A and Oraevsky A A 2003 Detection of ultrawide-band ultrasound pulses in optoacoustic tomography IEEE Trans. Ultrason. Ferroelectr. Freq. Control 50 1383-90

Burmistrova L V, Karabutov A, Rudenko O V and Cherepetskaya E B 1979 Influence of thermal nonlinearity on the thermooptical generation of sound Sov. Phys.-Acoust. 25 348-50

Clarke R L, Bush N L and Ter Haar G R 2003 The changes in acoustic attenuation due to in vitro heating Ultrasound Med. Biol. 29 127-35

Copland J A, Eghtedari M, Popov V L, Kotov N, Mamedova N, Motamedi M and Oraevsky A A 2004 Bioconjugated gold nanoparticles as a molecular based contrast agent: implications for imaging of deep tumors using optoacoustic tomography Mol. Imag. Biol. 6 341-9

Duck F A 1990 Physical Properties of Tissue (New York: Academic)

Esenaliev R O, Karabutov A A and Oraevsky A A 1999 Sensitivity of laser opto-acoustic imaging in detection of small deeply embedded tumors IEEE J. Sel. Top. Quantum Electron. 5 981-8

Esenaliev R O, Larina I V, Larin K V, Deyo D J, Motamedi M and Prough D S 2002 Optoacoustic technique for noninvasive monitoring of blood oxygenation: a feasibility study Appl. Opt. $414722-31$

Esenaliev R O, Petrov Y Y, Hartrumpf O, Deyo D J and Prough D S 2004 Continuous, noninvasive monitoring of total hemoglobin concentration by an optoacoustic technique Appl. Opt. $\mathbf{4 3}$ 3401-7

Gertner M R, Wilson B C and Sherar M D 1997 Ultrasound properties of liver tissue during heating Ultrasound Med. Biol. 23 1395-403 
Graham S J, Bronskill M J and Henkelman R M 1998 Time and temperature dependence of MR parameters during thermal coagulation of ex vivo rabbit muscle Magn. Reson. Med. 39 198-203

Gusev V E and Karabutov A 1993 Laser Optoacoustics (New York: AIP)

Karabutov A A, Savateeva E V and Oraevsky A A 2003 Optoacoustic tomography: new modality of laser diagnostic systems Laser Phys. 13 711-23

Kikoin I K 1976 Tables of Physical Parameters (Moscow: Atomizdat)

Kirillov A G, Lozhkarev V V, Mansfel'd A D, Reiman A M, Friedman G I and Yakovlev I V 1999 Application of quasi-resonant ultrasonic detectors in the optoacoustic tomography of biological objects Quantum Electron. 29 832-5

Larin K V, Larina I V, Motamedi M and Esenaliev R O 2002 Optoacoustic laser monitoring of cooling and freezing of tissues Quantum Electron. 32 953-8

Laufer J, Simpson R, Kohl M, Essenpreis M and Cope M 1998 Effect of temperature on the optical properties of ex vivo human dermis and subdermis Phys. Med. Biol. 43 2479-89

Lehmann J F 1990 Therapeutic Heat and Cold (Baltimore: Williams \& Wilkins)

MaassMoreno R and Damianou C A 1996 Noninvasive temperature estimation in tissue via ultrasound echo-shifts 1 . Analytical model J. Acoust. Soc. Am. 100 2514-21

Schule G, Huttmann G, Framme C, Roider O and Brinkmann R 2004 Noninvasive optoacoustic temperature determination at the fundus of the eye during laser irradiation J. Biomed. Opt. 9 173-9

Seip R and Ebbini E S 1995 Noninvasive estimation of tissue temperature response to heating fields using diagnostic ultrasound IEEE Trans. Biomed. Eng. 42 828-39
Seip R, VanBaren P, Cain C A and Ebbini E S 1996 Noninvasive real-time multipoint temperature control for ultrasound phased array treatments IEEE Trans. Ultrason. Ferroelectr. Freq. Control 43 1063-73

Sigrist M W 1986 Laser generation of acoustic waves in liquids and gases J. Appl. Phys. 60 R83-122

Steiner P, Botnar R, Dubno B, Zimmermann G G, Gazelle G S and Debatin J F 1998 Radio-frequency-induced thermoablation: monitoring with T1-weighted and proton-frequency-shift MR imaging in an interventional 0.5-T environment Radiol. 206 803-810

Wang L H V 2003 Ultrasound-mediated biophotonic imaging: a review of acousto-optical tomography and photo-acoustic tomography Disease Markers 19 123-38

Wang X D, Ku G, Wegiel M A, Bornhop D J, Stoica G and Wang L H V 2004 Noninvasive photoacoustic angiography of animal brains in vivo with near-infrared light and an optical contrast agent Opt. Lett. 29 730-2

Wang X D, Pang Y J, Ku G, Stoica G and Wang L H V 2003a Three-dimensional laser-induced photoacoustic tomography of mouse brain with the skin and skull intact Opt. Lett. 28 1739-41

Wang X D, Pang Y J, Ku G, Xie X Y, Stoica G and Wang L H V 2003b Noninvasive laser-induced photoacoustic tomography for structural and functional in vivo imaging of the brain Nature Biotechnol. 21 803-6

Wang X D, Xu Y, Xu M H, Yokoo S, Fry E S and Wang L H V 2002 Photoacoustic tomography of biological tissues with high cross-section resolution: reconstruction and experiment $\mathrm{Med}$. Phys. 29 2799-805

Weast R C 1974 Handbook of Chemistry and Physics 54 edn (Boca Raton, FC: CRC Press)

Welch A J and van Gemert M J C 1995 Optical-Thermal Response of Laser-Irradiated Tissue (New York: Plenum) 